\title{
Pelvic lymph node dissection and outcome of robot-assisted radical cystectomy for bladder carcinoma
}

\author{
Aldrin J. Gamboa $\cdot$ Jennifer L. Young • Atreya Dash • \\ Jose Benito Abraham • Geoffrey N. Box • \\ David K. Ornstein
}

Received: 9 October 2008 / Accepted: 8 January 2009 / Published online: 4 February 2009

(c) The Author(s) 2009. This article is published with open access at Springerlink.com

\begin{abstract}
Introduction Pelvic lymph node dissection (PLND) at the time of radical cystectomy for urothelial carcinoma of the bladder is critical for accurate staging and may improve oncologic outcomes. Minimally invasive approaches have been criticized for limiting the extent of the PLND. We reviewed our experience with PLND and its perioperative outcomes with robotassisted laparoscopic radical cystectomy (RARC).

Methods Data were collected prospectively from 50 patients scheduled for RARC. Included in the analysis were patients who had RARC and a standard PLND. The entire extirpative portion of the procedure was performed using the da Vinci Robot (Intuitive Surgical, Sunnyvale, CA, USA).

Results A total of 41 patients were included in the study: 30 men and 11 women with a mean age of 69.7 years
\end{abstract}

\footnotetext{
A. J. Gamboa · J. L. Young ( $₫)$ · A. Dash · J. B. Abraham Department of Urology, University of California, Irvine, 333 City Boulevard West, Suite 2100, Orange, CA 92868, USA

e-mail: jlyoung@uci.edu

A. J. Gamboa

e-mail: aldringamboa@yahoo.com

A. Dash

e-mail: dasha@uci.edu

J. B. Abraham

e-mail: Jose.Abraham@uth.tmc.edu

G. N. Box

Ohio State University, 540 Doan Hall,

410 W. 10th Ave Columbus, Columbus, OH 43210, USA

e-mail: Geoffrey.Box@ osumc.edu

D. K. Ornstein

Vanguard Urologic Institute, 6400 Fannin St.,

Suite 2300, Houston, TX 77030, USA

e-mail: David.Ornstein@vanguardurology.com
}

(range 49-85) and a mean body mass index of 26.9 (range 19.5-43.7). The median total operative time was $497.77 \mathrm{~min}$ (320-805). The mean estimated blood loss was $253.66 \mathrm{ml}$ (range 50-700). The transfusion rate was $44 \%$ (18 out of 41) ranging from 0 to 4 units (median 0 units of blood). The mean total number of lymph nodes retrieved was 25.07 (range 4-68). Nodal metastases were seen in $14.63 \%(6 / 41)$. Rate of positive surgical margin was $4.87 \%$ (2/41). The median length of hospital stay was 8 days (5-37). The median duration of nasogastric tube, time to ambulation, first clear liquid intake, passage of colonic gas, time to bowel movement, and start of solid food intake were $1(0-5), 2(1-7), 3$ (2-10), $4(1-6), 5(2-11)$ and 6 days (3-24), respectively.

Conclusion An adequate PLND, comparable with that recommended for open surgery, can be performed safely with robot assistance. The perioperative outcomes were likewise comparable with that of the gold standard, open cystectomy.

Keywords Robotic · Cystectomy · Minimally invasive · Lymphadenectomy $\cdot$ Laparoscopic

\author{
Abbreviations \\ PNLD Pelvic lymph node dissection \\ RARC Robot-assisted laparoscopic radical cystectomy \\ BMI Body mass index \\ ASA American Society of Anesthesiologists
}

\section{Introduction}

The American Cancer Society estimates that in 2007 there will have been 67,160 new cases of bladder cancer diagnosed in the USA and 13,750 deaths from this cancer [1]. 
While the majority of patients with bladder cancer present with superficial bladder tumors, about $20-40 \%$ will present with, or will progress to, muscle-invasive disease. Invasive disease is the potentially lethal form of bladder cancer and, if left untreated, more than $85 \%$ of patients will die from the disease within two years of diagnosis [2]. Despite an early and aggressive surgical approach toward high-grade, invasive bladder cancer, as many as $25 \%$ of patients are expected to have pathologic evidence of lymph node metastases at cystectomy [3]. Open radical cystectomy with standard bilateral pelvic iliac lymphadenectomy remains the gold standard treatment for high-grade, muscle-invasive bladder cancer [4-10].

Less invasive approaches to the surgical management of many urologic cancers have been shown to offer considerable benefits in terms of speed of recovery, blood loss, postoperative pain, and cosmetic results in the treatment of urologic malignancies [11-14]. There have now been several trials, albeit small and nonrandomized, that have shown a benefit for robotic cystectomy compared with open cystectomy with regard to less blood loss and short-term morbidity $[15,16]$. Because robotic cystectomy is a relatively new procedure, long-term oncologic efficacy remains to be determined. Several studies have demonstrated that the quality of surgery for radical cystectomy affects cancer control and overall survival $[17,18]$. One of the most important surgical factors is the extent of the lymphadenectomy [19]. For robotic radical cystectomy to be a viable procedure, it is essential that this "less invasive" procedure replicates the technical aspects of standard open procedures, for example PLND. The performance of an adequate pelvic lymph node dissection (PLND) has been regarded as one of the most significant challenges in replicating the open technique during robot-assisted radical cystectomy [20]. Here, we review our experience with robotic PLND and its perioperative outcomes as performed in conjunction with robot-assisted laparoscopic radical cystectomy (RARC) and compared these outcomes with existing surgical guidelines.

\section{Materials and methods}

Between September 2004 and May 2008, data were collected prospectively on 50 consecutive patients scheduled for robot-assisted cystectomy. Forty-one patients underwent a formal robotic PLND in conjunction with RARC. Excluded from the analysis were patients that had inability to tolerate pneumoperitoneum $(n=1)$, presence of grossly enlarged pelvic lymph node $(n=3)$, and limited PLND because of age and co-morbid conditions $(n=5)$.

The data collection protocol was approved by the University of California, Irvine Institutional Review Board.
Surgeries were performed by a single fellowship-trained, urologic oncologist, who had previously performed 110 robot-assisted laparoscopic radical prostatectomies prior to performing the initial robotic cystectomy in September 2004. The da Vinci S system was introduced in March 29, 2007 which slightly modified the technique in that the ports were all placed $2 \mathrm{~cm}$ higher than for the standard system. This facilitated completion of the upper extent of the lymph node dissection. Patient data analyzed were gender, age, body mass index, length of hospital stay, operative time, console time, estimated blood loss, blood transfusion rate, total number of lymph nodes harvested, rate of positive lymph nodes, rate of positive surgical margin, perioperative and immediate postoperative complications, length of hospital stay, duration of nasogastric tube, time to ambulation, time to first passage of flatus, time to first clear liquid intake, time to first bowel movement, and time to first solid intake.

\section{Technique}

All patient were prepared with a standard mechanical and antibiotic bowel regimen preoperatively and standard perioperative intravenous antibiotics. After induction of general anesthesia, patients were positioned in steep Trendelenberg with arms tucked and legs abducted and lowered on spreader bars to facilitate docking of the robot. A nasogastric tube was placed and an 18 French foley catheter was inserted on the sterile surgical field. The procedure was approached intraperitoneally following insufflation of the abdomen with carbon dioxide obtained using a Veress needle. The six-port configuration has been described previously [21, 22]. A $12 \mathrm{~mm}$ trocar for the camera was placed above the umbilicus. Two 8-mm ports for the robot arms were placed just lateral to the medial umbilical ligaments. A lateral 10/12-mm port on the right and an $8-\mathrm{mm}$ robotic port were placed medial and superior to the anterior superior iliac spine. A 5-mm port was placed in the upper right quadrant for the suction/irrigator instrument. The camera port was placed $24-25 \mathrm{~cm}$ from the symphysis pubis and the two robotic ports were placed $18-20 \mathrm{~cm}$ from the symphysis pubis. The third robotic port was placed in the anterior axillary line on the left side about the level of the umbilicus (Fig. 1). Similar port placement has been described by Hafron et al. [23]. PLND was performed either before or after completion of radical cystectomy. In these patients a standard lymph node dissection was performed in which the genitofemoral nerve was the lateral border, the obturator nerve was the posterior border, the bladder was the medial border, and the internal inguinal ring was the distal border. Proximally the dissection was carried above the hypogastric artery, typically $2 \mathrm{~cm}$ below 


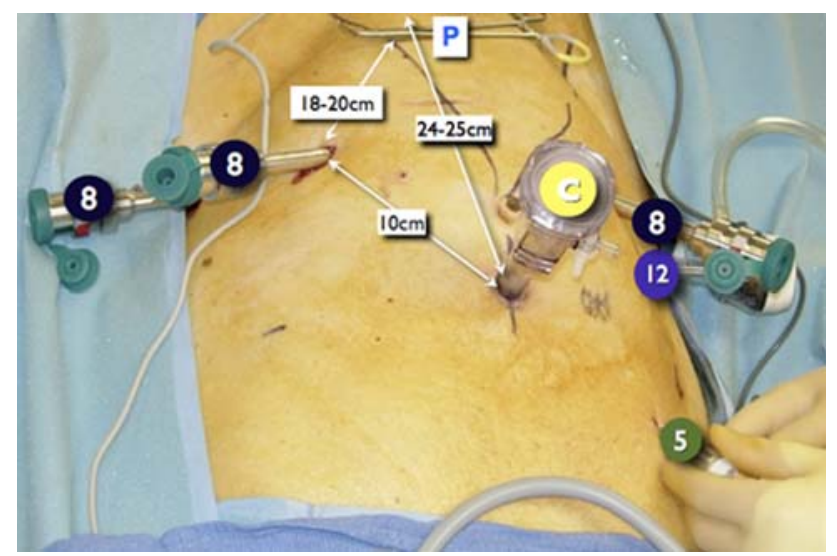

Fig. 1 Port placement during robot-assisted radical cystectomy with pelvic lymph node dissection. Symphysis pubis $(P)$; camera port $(C)$; $10 / 12 \mathrm{~mm}$ assistant port (12); robotic port (8); suction/irrigation port (5). Distance from camera to symphysis pubis is $24-25 \mathrm{~cm}$; distance from robotic port to symphysis pubis is $18-20 \mathrm{~cm}$

the bifurcation of the aorta and occasionally to the bifurcation of the aorta. Lymph nodes were typically collected as external iliac and obturator node packets on each side. Occasionally specimens were broken into common iliac, external iliac, and internal iliac. Occasionally presacral nodes were included.

\section{Results}

Forty-one patients were included in the study, 30 men and 11 women with a mean age of 69.7 years (range 49-85) and a mean body mass index of 26.9 (range 19.5-43.7)). Twenty-six patients had an American Society of Anesthesiologists (ASA) score of III, and 14 and 1 patients were classified as ASA II and IV, respectively. An ileal conduit or a continent urinary diversion was performed in 24 and 17 patients, respectively. The mean total operative time was 497.77 min (320-805) with a mean robotic console time of 267.32 min (range 200-387). The time to complete the PLND was approximately $90 \mathrm{~min}$. The mean estimated blood loss was $253.66 \mathrm{ml}$ (range 50-700). The transfusion rate was $44 \%$ (18 out of 41 ) with a range of $0-4$ units (median 0 units). The median total number of lymph nodes retrieved was 23 (range 4-68). Lymph nodes were typically collected as external iliac and obturator node packets on each side. Occasionally specimens were broken into common iliac, external iliac, and internal iliac. Occasionally presacral nodes were included. Nodal metastases were seen in $14.63 \%(6 / 41)$. Rate of positive surgical margin was $4.87 \%(2 / 41)$. The ratio of positive lymph nodes to the number harvested for the six patients with positive lymph nodes were $4 / 44,19 / 47,4 / 14,1 / 28,1 / 43$, and $3 / 23$, respectively. The rate of positive surgical margin was $4.87 \%(2 / 41)$ and both patients had stage pT4 disease on final pathology report. The extirpative portion of all the procedures was completed entirely intracorporeally. There were no conversions to open during the entire extirpative procedure. Intraoperative complications occurred in two patients and both were partial transections of the obturator nerve. These were repaired with interrupted 5-0 prolene sutures and the patients recovered without clinical sequelae. During lymphadenectomy one patient had abulsion of a branch of the common iliac vein, this was controlled robotically without significant blood loss. Perioperative complications related to the lymphadenectomy were seen in three patients: clinically significant lymphocele (1) and deep vein thrombosis (2). Other perioperative complications included ileus (4) and bowel obstruction requiring exploratory laparotomy and bowel resection (1). Three patients developed ureteroenteric stricture and one patient had bladder neck contracture. The median length of hospital stay was 8 days (5-37). The median duration of nasogastric tube, time to ambulation, first clear liquid intake, passage of colonic gas, time to bowel movement and start of solid food intake were $1(0-5)$, $2(1-7), 3$ (2-10), 4 (1-6), 5 (2-11), and 6 days (3-24), respectively. A comparison of the RARC patients' demographics and outcomes $(n=22)$ using the conventional da Vinci system compared with the da Vinci S system showed similar demographic and perioperative outcomes $(P>0.05)$. However, the entire extirpative time (console time) was reduced $(P=0.005)$ using the da Vinci S system.

\section{Discussion}

Open radical cystectomy with bilateral pelvic iliac lymphadenectomy remains the gold standard treatment for high-grade, muscle-invasive bladder cancer, because its benefits have been demonstrated by large clinical series with long follow up [4-10]. Stein and Skinner [5] showed that the pathological stage of the primary tumor and the presence of regional lymph node metastases were the most important determinants of survival in patients undergoing radical cystectomy for bladder cancer. The recurrence-free and overall survival for the entire 1,054 patients was $68 \%$ at 5 years and $66 \%$ at 10 years, with most deaths occurring within the first 3 years following radical cystectomy secondary to bladder cancer recurrences. For lymph nodenegative, organ-confined disease $(\mathrm{p} \leq \mathrm{pT} 2 \mathrm{~b}) 5$-year and 10 -year recurrence-free rates were 85 and $82 \%$, respectively. Herr et al. [10] showed that a thorough PLND, which increased the number of lymph nodes removed, affects bladder cancer outcomes. The five-year survival rate for patients with fewer than ten nodes retrieved was $44 \%$ compared with $61 \%$ for patients with more than ten nodes examined [6]. The introduction of laparoscopy has 
facilitated the application of minimally invasive surgery to the management of urologic cancers, and laparoscopic procedures have become, to some, the preferred approach to radical nephrectomy for most patients [24]. The application of laparoscopy to radical cystectomy has been far more limited. Parra et al. [25] reported the first laparoscopic simple cystectomy for pyocystis 15 years ago, and Sanchez et al. [26] reported the first laparoscopic cystectomy for bladder cancer with extracorporeal construction of an ileal conduit. The feasibility of completely intracorporeal urinary diversion was later shown by Gill et al. [27].

The potential benefits of the minimally invasive laparoscopic approaches include reduced length of hospital stay, reduced intraoperative blood loss, and shorter convalescence. We had a low threshold for transfusion in this report, because many of the patients had preoperative anemia, in addition to advanced age and multiple co-morbidities. Although the oncologic efficacy of RARC remains to be proven in larger studies, pathologic and short-term outcomes suggest that results are likely to be comparable with those of contemporary open radical cystectomy [28]. Although the feasibility of laparoscopic cystectomy has been demonstrated, its widespread acceptance has been limited because of the technical challenges associated with this procedure. The introduction of a robotic interface has dramatically expanded the application of minimally invasive surgery for radical prostatectomy [29] and it is likely to have the same impact on radical cystectomy. Initial series by Menon et al. [30] and Guru et al. [31] showed the feasibility of robot-assisted radical cystectomy in three and seven female patients, respectively. Recently, Guru et al. [32] showed that performance of RARC can be safely considered for patients that are candidates for open cystectomy. Robot-assisted surgery (da Vinci Surgical System, Intuitive Surgical, Sunnyvale, CA, USA) offers potential advantages over traditional surgical approaches by providing excellent three-dimensional vision, articulating instruments, ergonomic design, and tenfold magnification of the surgical field. Similar to what was seen with robotic prostatectomy, the advantages of robotics should shorten the learning curve associated with minimally invasive cystectomy as compared with standard laparoscopy, and should facilitate performance of an adequate pelvic lymphadenectomy.

Skinner et al. reviewed existing data today on the impact of surgical techniques on oncological outcomes. Its purpose was to encourage the surgeon to focus on surgical technique and emphasized the commitment to achieving complete surgical excision, minimizing local recurrence, and maximizing the removal of potentially involved lymph nodes [33]. The role of a regional lymphadenectomy in the surgical management of high-grade invasive transitional cell carcinoma of the bladder has evolved over the last several decades and is now commonly accepted [34]. Pelvic lymphadenectomy may provide a therapeutic benefit in addition to its prognostic information. Although, the absolute limits of the lymphadenectomy remain a subject of controversy, with no level I evidence, it is generally agreed that adequate lymph node dissection should extend up to or beyond the hypogastric artery and include the nodal packets medial to and below the obturator nerve [35], and that the extent of the PLND correlates with survival advantage [36]. Poulsen et al. [8] retrospectively compared extended pelvic lymphadenectomy (proximally to the aortic bifurcation) with limited dissection (proximally to the common iliac) at the time of open radical cystectomy, and found a statistically significant though small improvement in five-year recurrence-free survival for the extended dissection group (62 vs. 56\% limited dissection) and a substantial improvement ( 85 vs. 64\%) for the subgroups with tumors confined to the bladder wall (tumor stage pT3a or less) [8]. The Bladder Cancer Collaborative Group proposed guidelines for the surgical management of bladder. At least 10-14 lymph nodes should be retrieved at cystectomy, with a margin positive rate of less than $10 \%$ of all cases performed (less than $15 \%$ for bulky tumors and less than $20 \%$ for salvage cases) [37].

Pelvic lymph node dissection requires an additional 30 $60 \mathrm{~min}$ to the operative time, but no significant difference in perioperative mortality, early complications, or need for blood transfusions $[6,8]$. Since instituting titanium clips to secure all lymphatics, we have not had any lymphoceles. A survival advantage may result from a more complete excision of tissue harboring subclinical micrometastasis, as supported by polymerase chain reaction analysis revealing bladder cancer-associated DNA in the lymph nodes of 29$33 \%$ of patients classified as having node-negative disease by standard histopathology [38, 39]. Recently, lymph node density, even in the presence of positive lymph nodes, offers further prognostic value in predicting disease-specific survival [40]. Our results demonstrated an acceptable number of harvested lymph nodes and risk of positive surgical margins supporting our comparable assumption that oncologic standards for radical cystectomy can be maintained with RARC. These results confirm the technical feasibility of a robot-assisted cystectomy in the performance of a pelvic lymphadenectomy with a single surgical assistant using a six-port configuration.

In addition, the objective of performing minimally invasive surgery is to maintain comparable outcome while minimizing complications. As experience increases, further improvements in clinicopathologic and functional outcome can be achieved, as shown by the Badani et al. [41] after performing 2,776 cases of robotic radical prostatectomy. As more patients elect for minimally invasive procedures over conventional open surgery, we believe that experience with RARC may further improve clinical outcomes. 


\section{Conclusion}

An acceptable PLND can be performed safely with robot assistance, with outcome comparable with that achieved with the open approach. These data support the continued evaluation of RARC as a less invasive surgical approach to management of invasive bladder cancer. The oncologic efficacy needs to be determined in a much larger study with longer follow up. The perioperative outcomes were likewise comparable with that of the gold standard, open cystectomy. With experience, the extirpative time was observed to decrease.

Open Access This article is distributed under the terms of the Creative Commons Attribution Noncommercial License which permits any noncommercial use, distribution, and reproduction in any medium, provided the original author(s) and source are credited.

\section{References}

1. Jemal L, Siegel R, Ward E et al (2007) Cancer Statistics, 2007. Cancer J Clin 57:43-66

2. Prout G, Marshal V (1956) The prognostic with untreated bladder cancer. Cancer 9:551-558

3. Stein J, Skinner D (2006) Radical cystectomy for invasive bladder cancer: long term results of a standard procedure. World J Urol 24(3):296-304

4. Stein J (2003) Indications for early cystectomy. Urology 54:8-29

5. Stein J, Lieskovsky G, Cote R et al (2001) Radical cystectomy in the treatment of invasive bladder cancer: long-term results in 1,054 patients. J Clin Oncol 19:666-675

6. Herr H, Faulkner J, Grossman H et al (2004) Surgical factors influence bladder outcome: a cooperative group report. J Clin Oncol 22:2781-2789

7. Leissner J, Ghonei A, Abol-Enein H et al (2004) Extended radical lymphadenectomy in patients with urothelial bladder cancer: results of a prospective multicenter study. J Urol 171:139-144

8. Poulsen A, Horn T, Steven K (1998) Radical cystectomy: extending limits of pelvic lymph node dissection improves survival for patient with bladder cancer confined to the bladder wall. J Urol 160:215-220

9. Dalbagni G, Gnega E, Hashibe et al. (2001) Cystectomy for bladder cancer: contemporary series. J Urol 165: 1111-1116

10. Herr H, Bochner B, Dalbagni G et al (2002) Impact of the lymph nodes retrieved on outcome in patients with muscle invasive bladder cancer. J Urol 167:1295-1298

11. Hemal A, Kumar A, Kumar R et al (2007) Laparoscopic versus open radical nephrectomy for large renal tumors: a long term prospective comparison. J Urol 177:862-866

12. Roupret M, Huperton V, Sanderson K et al (2007) Oncologic control after open or laparoscopic nephroureterectomy for upper urinary tract transitional cell carcinoma: a single center experience. Urology 69(4):656-661

13. Miller J, Smith A, Kouba E et al (2007) Prospective evaluation of short term impact and recovery of health related quality of life in men undergoing robotic assisted radical prostatectomy versus open prostatectomy. J Urol 178:854-859

14. Raventos BR, Gomez LE, Cecchini RL et al (2007) Laparoscopic versus open radical prostatectomy. Actas Urol Esp 31(9):36353639
15. Wang G, Barocas D, Raman J et al (2008) Robotic versus open radical cystectomy: prospective comparison of perioperative outcomes and pathological measures of early oncological efficacy. BJU Int 101(1):89-93

16. Pruthi R, Wallen E (2007) Robotic assisted laparoscopic radical cystoprostatectomy: operative and pathological outcomes. J Urol 178(3):814-818

17. Skinner E, Stein J, Skinner D (2007) Surgical benchmarks for the treatment of invasive bladder cancer. Urol Oncol 25(1):66-71

18. Herr H (2005) Surgical factors in the treatment of superficial and invasive bladder cancer. Urol Clin N Am 32:157-164

19. Dhar N, Klein E, Reuther A et al (2008) Outcome after radical cystectomy with limited or extended pelvic lymph node dissection. J Urol 179:873-878

20. Gettman M, Blute M, Peschel R (2003) Current status of robotics in urologic laparoscopy. Eur Urol 4(2):106-112

21. Sala L, Matsunaga G, Corica F et al (2006) Robot-assisted laparoscopic radical cystoprostatectomy and totally intracorporeal ileal neobladder. J Endourol 20(4):233-235

22. Abraham J, Young J, Box G et al (2007) Comparative analysis of laparoscopic and robot-assisted radical cystectomy with ileal conduit urinary diversion. J Endourol 21(12):1473-1480

23. Hafron J, Kaouk JH, Haber GP et al. (2007) Extended pelvic lymphadenectomy during robotic cystectomy. Eng Urol Soc (Abstract 114.)

24. Eskicorapci S, Teber D, Schulze M et al (2007) Laparoscopic radical nephrectomy: the new gold standard surgical treatment for localized renal cell carcinoma. Scientific World J 7:825-836

25. Parra R, Andrews C, Jones J et al (1992) Laparoscopic cystectomy: initial report on a new treatment for the retained bladder. J Urol 48:1140-1144

26. Sanchez B, Gallego P, Reche R et al (1995) Laparoscopic cystectomy and ileal conduit: a case report. J Endourol 9(1):59-62

27. Gill I, Fergany A, Klein E et al (2000) Laparoscopic radical cystoprostatectomy with ileal conduit performed completely intracorporeal: the initial 2 cases. Urology 56(1):26-29

28. Haber GP, Gill IS (2007) Laparoscopic radical cystectomy for cancer: oncological outcomes at up to 5 years. BJU Int 100:137142

29. Yee D, Ahlering T (2007) Radical prostatectomy: a current perspective. J Urol 178(2):376-377

30. Menon M, Hemal A, Tewari A et al (2004) Robot-assisted radical cystectomy and urinary diversion in female patients: technique with preservation of the uterus and vagina. J Am Coll Surg 198(3):386-393

31. Guru K, Nogueira M, Piacente P et al (2007) Robot-assisted anterior exenteration: technique and initial series. $\mathrm{J}$ Endourol 21(6):633-639

32. Guru K, Kim H, Piacente P et al (2007) Robot-assisted radical cystectomy: initial experience at Roswell Park Cancer Institute. Urology 69(3):469-474

33. Skinner E, Stein J, Skinner D (2007) Surgical benchmarks for the treatment of invasive bladder cancer. Urol Oncol 25(1):66-71

34. Stein J, Skinner D (2007) The role of lymphadenectomy in high grade invasive bladder cancer. Urol Clin N Am 32:187-197

35. Stein JP (2006) Lymphadenectomy in bladder cancer: how high is "high enough"? Urol Oncol 24:349-355

36. Konety B, Joslyn S, O’Donell M (2003) Extent of pelvic lymphadenectomy and its impact on outcome in patients diagnosed with bladder cancer: analysis of data from the surveillance, epidemiology and end results program data base. J Urol 169:946950

37. Herr H, Lee C, Chang S et al (2004) Standardization of radical cystectomy and pelvic lymph node dissection for bladder cancer: a collaborative group report. J Urol 171:1823-1828 
38. Retz M, Lehmann J, Szysnih C et al (2004) Detection of occult tumor cells in lymph nodes from bladder cancer patients by $\mathrm{MuC7}$ nested RT-PCK. Eur Urol 45:314-319

39. Copp H, Chin J, Conany M et al (2006) Prospective evaluation of the prognostic relevance of molecular staging for urothelial carcinoma. Cancer 107:60-66

40. Kassouf W, Agarwal P, Herr H et al (2008) Lymph node density is superior to TNM nodal status in predicting disease-specific survival after radical cystectomy for bladder cancer: analysis of pooled data from MDACC and MSKCC. J Clin Oncol 26(1):121126

41. Badani K, Kaul S, Menon M (2007) Evolution of robotic radical prostatectomy: assessment after 2766 procedures. Cancer 110(9):1951-1958 